\title{
ESCOAMENTO DA ÁGUA DA CHUVA PELO TRONCO DAS ÁRVORES EM UMA FLORESTA ESTACIONAL SEMIDECIDUAL ${ }^{1}$
}

\begin{abstract}
Alexandre Simões Lorenzon ${ }^{2}$, Herly Carlos Teixeira Dias ${ }^{3}$ e Kelly Cristina Tonello ${ }^{4}$
RESUMO - Este trabalho foi desenvolvido na Estação de Pesquisas, Treinamento e Educação Ambiental Mata do Paraíso, situada no Município de Viçosa, na Zona da Mata de Minas Gerais, Brasil, e teve como objetivo avaliar o escoamento pelo tronco em um fragmento de Mata Atlântica, no período de agosto de 2009 a março de 2011. Para quantificar esse escoamento, foram demarcadas seis parcelas de $10 \times 10 \mathrm{~m}$, sendo em cada parcela adaptados coletores à base de poliuretano nos troncos das árvores com circunferência $15,0 \mathrm{~cm}$. Um pluviômetro foi instalado em local aberto para quantificar a água diretamente da chuva. Além disso, realizou-se uma análise qualitativa dos indivíduos do escoamento pelo troco, avaliando a qualidade do fuste e da copa, posição no estrato da floresta e infestação de cipó. Em todo o período de análises, foram realizadas 75 medições. Nas parcelas de escoamento pelo tronco foram amostrados 126 indivíduos, distribuídos em 29 famílias e 59 espécies. A precipitação em aberto foi de $2.391,63 \mathrm{~mm}$, e o escoamento pelo tronco somou 31,59 mm, ou $1,32 \%$ da precipitação em aberto. A espécie com maior escoamento pelo tronco foi a Euterpe edulis, com um volume médio de água escoado de 637,00 L. Das espécies com maior escoamento pelo tronco, 73,91\% encontravam-se no estrato inferior da floresta, evidenciando que algumas espécies possuem algum tipo de adaptação morfológica para captação da água da chuva.
\end{abstract}

Palavras-chave: Mata Atlântica; Hidrologia florestal; Escoamento pelo tronco.

\section{STORMWATER RUNOFF ALONG THE TREE TRUNK IN A SEASONAL SEMIDECIDUOUS FOREST}

\begin{abstract}
This study was conducted at the Estação de Pesquisas, Treinamento e Educação Ambiental Mata do Paraíso, located in Viçosa, Minas Gerais, Brazil, and its goal is to evaluate the stem flow of the trees of a fragment of Atlantic Forest, from August 2009 to March 2011. In order to quantify the stem flow six plots were demarcated, measuring $10 \mathrm{~m} \times 10 \mathrm{~m}$. In each plot polyurethane based collectors were adapted on the stem of the tree with circumference $15.0 \mathrm{~cm}$. A rain gauge was installed in an open area to quantify the water directly from the rain. In addition, a qualitative analysis of the trees was performed regarding the stem flow, evaluating the quality of the stem and canopy, its location inside the forest and the level of lianas infestation. Considering the whole period of analysis, 75 measurements were performed. In the plots where the stem flow was quantified, 126 trees were sampled, distributed in 29 families and 59 species. The gross precipitation reached $2391.63 \mathrm{~mm}$ and the stem flow represented $31.59 \mathrm{~mm}$, or $1.32 \%$ of the gross precipitation. The species with the greater stem flow was Euterpe edulis, with a stem flow average of 637.00 liters. Among the species with the greater stem flow, $73.91 \%$ are located in the lower stratum of the forest, showing that some species have a type of morphological adaptation to capture rainwater.
\end{abstract}

Keywords: Atlantic Forest; Forest hydrology; Stem flow.

\footnotetext{
${ }^{1}$ Recebido em 11.12.2012 aceito para publicação em 07.04.2015.

${ }^{2}$ Universidade Federal de Viçosa, Programa de Pós-Graduação em Ciência Florestal, Viçosa, MG, Brasil. E-mail: <alelorenzon@yahoo.com.br>.

${ }^{3}$ Universidade Federal de Viçosa, Departamento de Engenharia Florestal, Viçosa, MG, Brasil. E-mail: <herly@ufv.br>.

${ }^{4}$ Universidade Federal de São Carlos, Departamento de Ciências Ambientais, Sorocaba, SP, Brasil. E-mail: <kellytonello@ufscar.br>.
} 


\section{INTRODUÇÃO}

Em florestas, parte da água da chuva que chega ao solo é originária do escoamento pelo tronco e, embora corresponda à pequena porcentagem da precipitação incidente acima do dossel, é de fundamental importância para muitas espécies da flora e para o ambiente.

O escoamento pelo tronco é o nome dado à fração da chuva temporariamente retida pela copa das árvores juntamente com aquela que atinge diretamente os troncos e, posteriormente, escoa pelo tronco das árvores, chegando ao solo (OLIVEIRA JUNIOR; DIAS, 2005). Essa via pode corresponder a $15 \%$ do total precipitado (TUCCI, 2001).

Alguns autores relataram que a distribuição localizada, resultante do escoamento pelo tronco, é significativa ao redor dos troncos e funciona como mecanismo de autoabastecimento que exerce efeito sobre a qualidade e quantidade de água que chega ao solo (PRICE, 1982; HUBER; OYARZÚN, 1983; JOHNSON, 1990).

Segundo Návare Bryan (1990), o volume recebido nas proximidades dos troncos de algumas espécies chega a ser cinco vezes superior àquele recebido por áreas mais distantes. Algumas espécies, por dependerem de muita umidade no solo ou por terem raízes que alcancem pouca profundidade, podem ter-se adaptado morfologicamente a captar a água da chuva pela copa, como forma de aumentar a disponibilidade de água próxima às raízes, principalmente nos períodos mais secos do ano.

Kellman e Roulet (1990), em estudo de escoamento pelo tronco em uma floresta tropical seca no México, observaram que árvores menores e arbustos do estrato inferior são mais eficazes na produção de escoamento pelo tronco que espécies do dossel superior e que o escoamento pelo tronco produzido por espécies do estrato inferior pode ser 10 vezes superior ao produzido por árvores do estrato superior.

Vários fatores podem interferir no escoamento pelo tronco, como: intensidade, ângulo e duração das chuvas, intervalo entre precipitações, densidade e estratificação das copas, diversidade e idade das espécies, filotaxia e tipo de folha, tamanho e forma do limbo, característica da casca e metodologia de avaliação (CROCKFORD; RICHARDSON, 1987; LIMA; LEOPOLDO, 1999; OLIVEIRA JUNIOR, 2006). Para Návar e Bryan (1990), variações no escoamento pelo tronco podem ser explicadas pela posição e ângulo dos ramos, sugerindo que árvores que apresentam galhos direcionados para cima produzem mais escoamento pelo tronco que árvores com galhos direcionados para baixo ou na horizontal.

Desse modo, este estudo objetivou avaliar o escoamento pelo tronco de indivíduos arbóreos em fragmento de Mata Atlântica no Município de Viçosa, MG, no período de agosto de 2009 a março de 2011.

\section{MATERIAL E MÉTODOS}

\section{1. Área de estudo}

Este trabalho foi desenvolvido na Estação de Pesquisas, Treinamento e Educação Ambiental Mata do Paraíso, pertencente à Universidade Federal de Viçosa, situada no Município de Viçosa, na Zona da Mata de Minas Gerais, a $229 \mathrm{~km}$ da capital Belo Horizonte. A área possui 194 ha e está localizada entre as latitudes de $20^{\circ} 41^{\prime} 20^{\prime \prime} \mathrm{S}$ e $20^{\circ} 49^{\prime} 35^{\prime}$ 'S e entre as longitudes de 42॰49'36"WGre 42 54'27"WGr (OLIVEIRA JUNIOR, 2006), a uma altitude média de 650 m (Figura 1).

De acordo com a classificação de Köppen, o clima da região é classificado como temperado quente, com verões chuvosos e invernos frios e secos (Cwb). A precipitação média anual e a umidade relativa ficam em torno de $1.268,2 \mathrm{~mm}$ e $81 \%$, respectivamente. A temperatura média anual é igual a $20^{\circ} \mathrm{C}$, conforme dados obtidos na estação meteorológica local, no período de 1968 a 2010.

A Estação de Pesquisas, Treinamento e Educação Ambiental Mata do Paraíso, pertencente ao domínio da Floresta Estacional Semidecidual, dentro do bioma denominado Floresta Tropical Atlântica ou Mata Atlântica (VELOSO et al., 1991), compõe um mosaico em diferentes estágios sucessionais e pequenas áreas de brejo (SILVA JUNIOR et al., 2004). Conforme a espécie florestal, poderá haver variação na queda das folhas, de meados de maio até praticamente fins de outubro (CASTRO et al., 1983).

Os solos da Mata do Paraíso são classificados como Latossolo Vermelho-Amarelo distrófico nas áreas com perfis convexos, Câmbicos nos topos das elevações em função da existência de horizonte $\mathrm{B}$ de pequena espessura, Argilossolos nas áreas de perfis côncavos e nos terraços e hidromórficos aluviais no leito maior (CORREA, 1984).

Revista Árvore, Viçosa-MG, v.39, n.3, p.423-430, 2015 


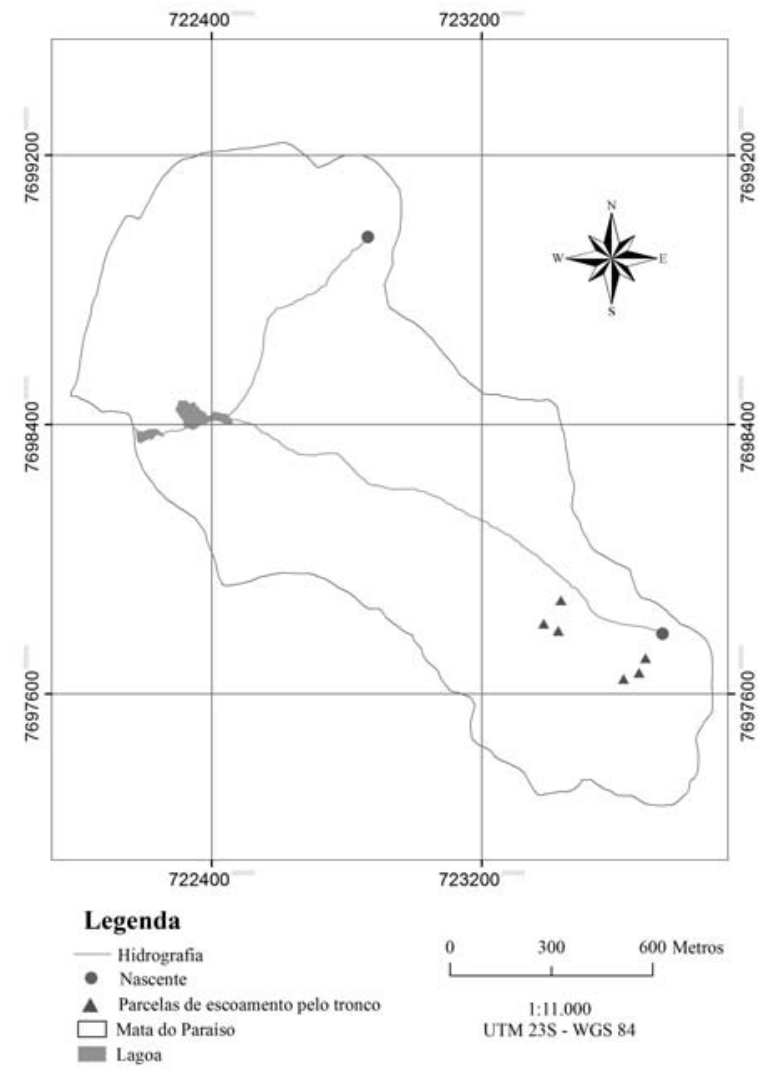

Figura 1 - Distribuição das parcelas experimentais de escoamento pelo tronco. Mata do Paraíso, ViçosaMG, 2009-2011.

Figure 1 - Distribution of experimental plots of stem flow. Mata do Paraíso, Viçosa-MG, 2009-2011.

\subsection{Metodologia}

Os dados de precipitação em aberto e escoamento pelo tronco foram obtidos no período de agosto de 2009 a março de 2011. As leituras foram feitas, quando possível, logo após cada evento de chuva. Assim, cada coleta é constituída de uma ou mais precipitações. As medições foram procedidas com o auxílio de provetas e baldes graduados.

\subsection{Precipitação em aberto (PA)}

A precipitação em aberto (PA) foi obtida por medições realizadas em pluviômetro simples de PVC, com área de captação de $167 \mathrm{~cm}^{2}$, instalado em uma torre acima do dossel da floresta e cujo cálculo foi realizado de acordo com a equação 1 . em que PA é a precipitação em aberto ( $\mathrm{mm}), \mathrm{V}$ é o volume do pluviômetro $(\mathrm{ml})$ e A é a área de captação do pluviômetro $\left(\mathrm{cm}^{2}\right)$.

\subsection{Escoamento pelo tronco (Et)}

Para quantificar o escoamento pelo tronco (Et), foram demarcadas seis parcelas de $10 \times 10 \mathrm{~m}$ (Figura 1). Em cada parcela foram adaptados coletores à base de poliuretano nos troncos das árvores com circunferência $\geq 15,0 \mathrm{~cm}$. Aágua da chuva foi direcionada por uma mangueira de 5/8" afixada aos coletores, para recipientes individuais de plásticos com capacidade para até $25 \mathrm{~L}$.

\subsection{Levantamento florístico}

O levantamento florístico foi realizado nas seis parcelas de escoamento pelo tronco. Todos os indivíduos com circunferência a $1,30 \mathrm{~m}$ do solo (CAP) $15 \mathrm{~cm}$ foram identificados. O material botânico foi identificado por meio de consultas ao Herbário da Dendrologia da Universidade Federal de Viçosa e por especialistas em identificação de plantas. As espécies foram classificadas em famílias, de acordo com o sistema do Angiosperm Phylogeny Group (APG III, 2009).

\subsection{Análise qualitativa dos indivíduos do escoamento pelo tronco}

A análise qualitativa foi realizada em todos os indivíduos do escoamento pelo tronco, através de observações visuais. Os seguintes parâmetros foram analisados:

Qualidade do fuste: 1 - Bom (tronco retilíneo e sem defeito aparente); 2 - Regular (tronco com alguma tortuosidade e poucos defeitos aparentes); e 3 - Inferior (tronco com muitas tortuosidades e defeitos aparentes).

Qualidade da copa: 1 - Boa (copa inteira e bem distribuída em torno do eixo); 2 - Regular (alguns galhos quebrados e com distribuição um pouco irregular); e 3 - Inferior (copa com metade dos galhos quebrados e, ou, distribuída em metade de seu eixo e, ou, inferior a $1 \mathrm{~m}^{2}$ na horizontal).

Estrato: 1 - Superior (com iluminação total); 2 -Médio (com iluminação parcial); e 3 - Inferior (sombra).

Revista Árvore, Viçosa-MG, v.39, n.3, p.423-430, 2015 
Infestação de cipó: 1 - Nenhum cipó; 2 - Cipó somente no tronco; 3 - Cipó somente na copa; e 4 - Cipó no tronco e na copa.

\section{RESULTADOS}

Em todo o período analisado, foram realizadas 75 medições, com precipitação em aberto acumulada igual a 2.391,6 mm. Nas parcelas de escoamento pelo tronco, foram amostrados 126 indivíduos distribuídos em 29 famílias e 59 espécies. Dos 15 indivíduos com maior escoamento pelo tronco, nove foram Euterpe edulis, três foram Sorocea bonplandii e os outros três foram Bathysa meridionalis, não identificada 3 e Zanthoxylum rhoifolium (Tabela 1). Os quatro indivíduos com maior escoamento pelo tronco pertencem à espécie Euterpe edulis, e para alguns desses indivíduos o volume total escoado pelo tronco, durante todo o período de estudos, chegou a ultrapassar 1.000 L de água.

$\mathrm{Na}$ Tabela 2 são apresentados os valores médios de escoamento pelo tronco por espécie. Percebe-se que a Euterpe edulis, não identificada 3, Zanthoxylum rhoifolium e Sorocea bonplandii foram as quatro espécies com maior escoamento pelo tronco. Com exceção da Bathys ameridionalis, os 15 indivíduos com maior escoamento pelo tronco estão representados por essas quatro espécies. Contudo, a E. edulis foi a espécie mais eficiente na captação da água da chuva.
Na Figura 2 está representada a análise qualitativa das 15 espécies com maior escoamento pelo tronco. Mais de $80,0 \%$ dessas espécies possuem troncos bons e regulares, $97,0 \%$ das espécies estão no estrato inferior e médio da floresta e 55,0\% não apresentam qualquer tipo de infestação de cipós.

\section{DISCUSSÃO}

Devido à capacidade limitada dos recipientes de armazenamento da água do escoamento pelo tronco - restritas no máximo a $25 \mathrm{~L}$-, a maioria dos recipientes transbordou com chuvas superiores a $50,0 \mathrm{~mm}$. No caso da E. edulis, esses recipientes chegaram a transbordar com chuvas de 22,0 mm. Assim, alguns valores foram subestimados, sugerindo que o potencial de algumas espécies na captação de água pode ser muito maior.

Em campo, foi observado que as E. edulis localizadas debaixo de outras de mesma espécie tiveram escoamento pelo tronco muito inferior ao das E. edulis de cima, assim como foi observado que os pluviômetros de precipitação interna, localizados debaixo das E. edulis, coletavam um volume muito menor de água que os demais pluviômetros. Germer et al. (2006), em estudo da precipitação interna na Amazônia, relataram a influência da palmeira Orbignya phalerata (babaçu), mesma família da Euterpe edulis, na redistribuição de água da chuva na floresta. Esses autores ressaltaram a capacidade natural das Orbignya phalerata em captar

Tabela 1 - Lista dos 15 indivíduos com maior escoamento pelo tronco (Et) medido ao longo do período de estudo. Mata do Paraíso, Viçosa-MG, 2009-2011.

Table 1 - List of 15 individuals with greater stem flow (Sf) measured throughout the study period. Mata do Paraíso, Viçosa-MG, 2009-2011.

\begin{tabular}{clc}
\hline $\mathrm{N}^{\mathrm{o}}$ & \multicolumn{1}{c}{ Nome científico } & Família \\
\hline 1 & Euterpe edulis Mart. & Arecaceae \\
2 & Euterpe edulis Mart. & Arecaceae \\
3 & Euterpe edulis Mart. & Arecaceae \\
4 & Euterpe edulis Mart. & Arecaceae \\
5 & Sorocea bonplandii (Baillon.) W. C. Burger & Moraceae \\
6 & Sorocea bonplandii (Baillon.) W. C. Burger & Moraceae \\
7 & Euterpe edulis Mart. & Arecaceae \\
8 & Bathys ameridionalis L.B.Sm. e Downs & Rubiaceae \\
9 & Não identificada 3 & Myrtaceae \\
10 & Euterpe edulis Mart. & Arecaceae \\
11 & Euterpe edulis Mart. & Arecaceae \\
12 & Euterpe edulis Mart. & Arecaceae \\
13 & Euterpe edulis Mart. & Arecaceae \\
14 & Sorocea bon plandii (Baillon) W. C. Burger & 647,26 \\
15 & Zanthoxylum rhoifolium Lam. & Moraceae \\
& & 635,63 \\
\end{tabular}

Revista Árvore, Viçosa-MG, v.39, n.3, p.423-430, 2015 
Escoamento da água da chuva pelo tronco...

Tabela 2 - Volume médio de escoamento pelo tronco (Et) das espécie amostradas na Mata do Paraíso, Viçosa-MG, $2009-2011$. Table 2 - Average volume of stem flow (Sf) of the species sampled in Mata do Paraíso, Viçosa-MG, 2009-2011.

\begin{tabular}{|c|c|c|c|c|}
\hline $\mathrm{N}^{\mathrm{o}}$ & Espécie & Família & Frequência & Et (L) \\
\hline 1 & Euterpe edulis Mart. & Arecaceae & 10 & 637,0 \\
\hline 2 & Não identificada 3 & Myrtaceae & 1 & 631,8 \\
\hline 3 & Zanthoxylum rhoifolium Lam. & Rutaceae & 1 & 360,6 \\
\hline 4 & Sorocea bonplandii (Baillon.) W. C. Burger & Moraceae & 8 & 272,6 \\
\hline 5 & Coffea arabica L. & Rubiaceae & 1 & 223,6 \\
\hline 6 & Allophylus edulis (A St.-Hil., Cambess. e A Juss) & Sapindaceae & 5 & 220,6 \\
\hline 7 & Cabralea canjerana (Vell.) Mart. & Meliaceae & 1 & 212,4 \\
\hline 8 & Psycotria sp. & Rubiaceae & 1 & 195,0 \\
\hline 9 & Bathys ameridionalis L. B. Sm. e Downs & Rubiaceae & 6 & 178,2 \\
\hline 10 & Ocotea corymbosa (Meisn.) Mez & Lauraceae & 1 & 157,5 \\
\hline 11 & Beilschmiedia sp. & Lauraceae & 5 & 153,4 \\
\hline 12 & Nectandra sp. & Lauraceae & 1 & 141,9 \\
\hline 13 & Myrcia sp. & Myrtaceae & 1 & 136,5 \\
\hline 14 & Jacaranda macrantha Cham. & Bignoniaceae & 1 & 133,6 \\
\hline 15 & Pouteria sp & Sapotaceae & 1 & 117,4 \\
\hline 16 & Pterigota brasiliensis Fr. All. & Malvaceae & 2 & 106,0 \\
\hline 17 & Sapium glandulatum (Vell.) Pax. & Euphorbiaceae & 2 & 98,8 \\
\hline 18 & Cecropia glaziovi Snethlage & Urticaceae & 1 & 96,2 \\
\hline 19 & Alchorneatrip linervia (Spreng.) M. Arg. & Euphorbiaceae & 2 & 92,0 \\
\hline 20 & Cupania vernalis Cambess. & Sapindaceae & 2 & 85,7 \\
\hline 21 & Taberna montana salzmannii A. DC. & Apocynaceae & 4 & 84,9 \\
\hline 22 & Pouteria ramiflora (Mart.) Radlk. & Sapotaceae & 1 & 76,7 \\
\hline 23 & Chorisia speciosa A. St.-Hil. & Malvaceae & 1 & 75,9 \\
\hline 24 & Guapira opposita (Vell.) Reitz & Nyctaginaceae & 9 & 75,8 \\
\hline 25 & Myrcia guajavaefolia O. Berg. & Myrtaceae & 1 & 75,4 \\
\hline 26 & Sebastiania commersoniana (Baill.) L.B. Sm. e Downs & Euphorbiaceae & 1 & 72,1 \\
\hline 27 & Cupania sp. & Sapindaceae & 1 & 70,0 \\
\hline 28 & Lonchocarpus muehlbergianus Hass1. & Fabaceae & 1 & 66,3 \\
\hline 29 & Aspidosperma olivaceumMüll. Arg. & Apocinaceae & 1 & 65,4 \\
\hline 30 & Campomanesia sp. & Myrtaceae & 7 & 61,3 \\
\hline 31 & Prunussellowii Koehne & Rosaceae & 1 & 61,2 \\
\hline 32 & Piptadenia gonoacantha (Mart.) J. F. Macbr. & Fabaceae & 2 & 52,1 \\
\hline 33 & Eugenea sp. & Myrtaceae & 1 & 51,3 \\
\hline 34 & Ocotea brachybotrya (Meisn.) Mez & Lauraceae & 2 & 50,7 \\
\hline 35 & Trattinickia rhoifoliaWilld. & Burseraceae & 4 & 50,2 \\
\hline 36 & Chrysophyllum sp. & Sapotaceae & 1 & 46,9 \\
\hline 37 & Solanum leucodendron Sendtn & Solanaceae & 2 & 46,5 \\
\hline 38 & Siparuna guianensis Aubl. & Siparunaceae & 2 & 45,0 \\
\hline 39 & Ilex brevicuspis Reissek & Aquifoliaceae & 1 & 44,9 \\
\hline 40 & Não identificada 2 & Lauraceae & 1 & 44,8 \\
\hline 41 & Sloanea sp & Elaeocarpaceae & 1 & 40,9 \\
\hline 42 & Allophylus semidentatus (Miq.) Radlk. & Sapindaceae & 1 & 40,6 \\
\hline 43 & Casearia lasiophylla Eichler & Salicaceae & 5 & 40,5 \\
\hline 44 & Machaerium nictitans (Vell.) Benth. & Fabaceae & 1 & 37,9 \\
\hline 45 & Não identificada 4 & Rubiaceae & 1 & 37,1 \\
\hline 46 & Didymopanax morototoni (Aubl.) Decnee Planch & Araliaceae & 1 & 37,0 \\
\hline 47 & Erythroxylum pelleterianum A St.-Hil. & Erythroxylaceae & 1 & 35,8 \\
\hline 48 & Não identificada 1 & Euphorbiaceae & 1 & 32,7 \\
\hline 49 & Nectandrarigida Ness & Lauraceae & 2 & 32,6 \\
\hline 50 & Guarea macrophyllaVahl. ssp. tuberculata & Meliaceae & 1 & 31,9 \\
\hline 51 & Guatteria nigrescens Mart. ST & Meliaceae & 1 & 31,6 \\
\hline 52 & Protium heptaphyllum (Aubl.) Marchand & Burseraceae & 1 & 29,6 \\
\hline 53 & Nectandra lanceolata Nesse Mart. ex Ness & Lauraceae & 4 & 28,8 \\
\hline 54 & Ingaedulis Mart. & Fabaceae & 2 & 25,5 \\
\hline 55 & Belangera glabra Cambess & Cunoniaceae & 1 & 25,1 \\
\hline 56 & Cybista xantisyphilitica (Mart.) ex DC. & Bignoniaceae & 1 & 24,1 \\
\hline 57 & Apuleia leiocarpa (Vogel) J. F. Macbr. & Fabaceae & 1 & 23.0 \\
\hline 58 & Rollinia sylvatica (A. St.-Hil.) Mart. & Annonaceae & 2 & 22,4 \\
\hline 59 & Carinia nalegalis (Mart.) Kuntze & Lecythidaceae & 1 & 19,1 \\
\hline
\end{tabular}


Qualidade do fuste

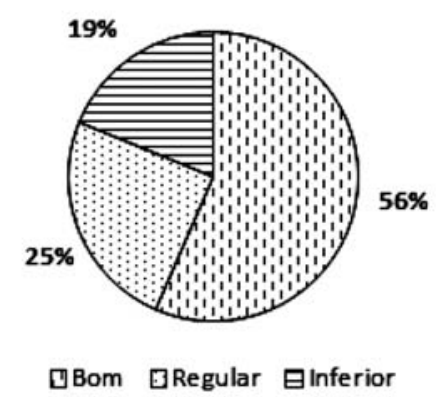

Estrato

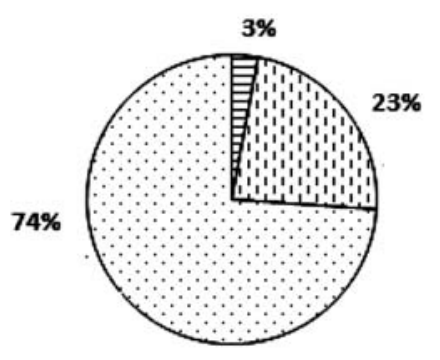

目Superior [MMédio IInferior

\section{Qualidade da copa}

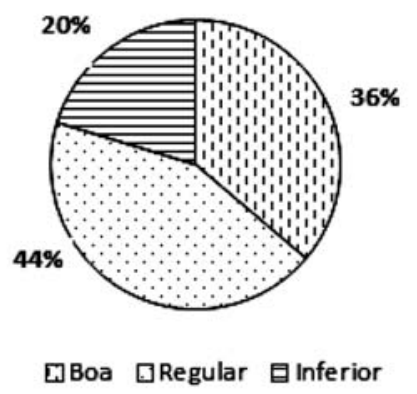

\section{Infestação de cipó}

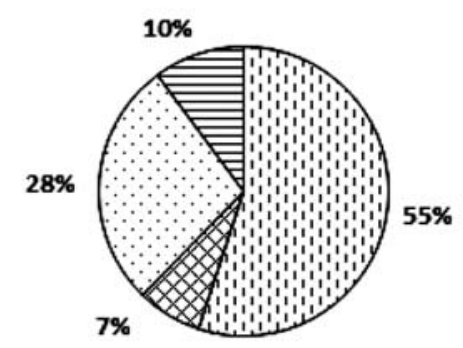

QNenhum \Cipó somente no tronco

Figura 2 - Análise qualitativa das 15 espécies com maior escoamento pelo tronco amostrado na Mata do Paraíso, ViçosaMG, 2009-2011.

Figure 2 - Qualitative analysis of 15 species with greater stem flow sampled in Mata do Paraíso, Viçosa-MG, $2009-2011$.

a água da chuva, em função do formato das folhas que atuam como calha, direcionando a água para pontos de gotejamento ou para o tronco. Isso indica que características individuais das espécies ou, mesmo, da família podem exercer influência significativa na quantidade de água escoada pelo tronco.

Contrariamente ao esperado, as espécies com maior escoamento pelo tronco estão no estrato inferior e médio da floresta, por isso é de se esperar a baixa infestação de cipós nesses indivíduos, haja vista o sombreamento delas. Não existe padrão com relação à qualidade da copa, o que indica que esse atributo não é determinante para o escoamento pelo tronco (Figura 2). Em contraponto, o fuste mais retilíneo tem influência positiva no escoamento pelo tronco.

Vários autores (LLOYD; MARQUES, 1988; NÁVAR; BRYAN, 1990; MANFROI et al., 2004) verificaram que árvores do sub-bosque produzem mais escoamento pelo tronco que árvores emergentes e com maior DAP. Isso pode ser indicativo que algumas espécies evoluíram morfologicamente na captação da água da chuva como forma de direcionar para suas raízes volume maior de água. Para algumas espécies, como E. edulis, que necessita de muita umidade no solo, essa adaptação evolutiva pode ser a forma encontrada para sobreviver em locais mais secos na floresta, podendo ainda explicar sua distribuição no ambiente.

A Figura 3 mostra que o escoamento pelo tronco da E. edulis possui alta correlação com a precipitação em aberto, como indica o modelo de regressão linear. Observa-se ainda que o coeficiente de determinação foi igual a $\mathrm{R}^{2}=0,8050$. Contudo, é provável que o coeficiente de determinação fosse mais próximo de 1 , caso os recipientes de armazenamento do escoamento pelo tronco não tivessem transbordado.

Revista Árvore, Viçosa-MG, v.39, n.3, p.423-430, 2015 


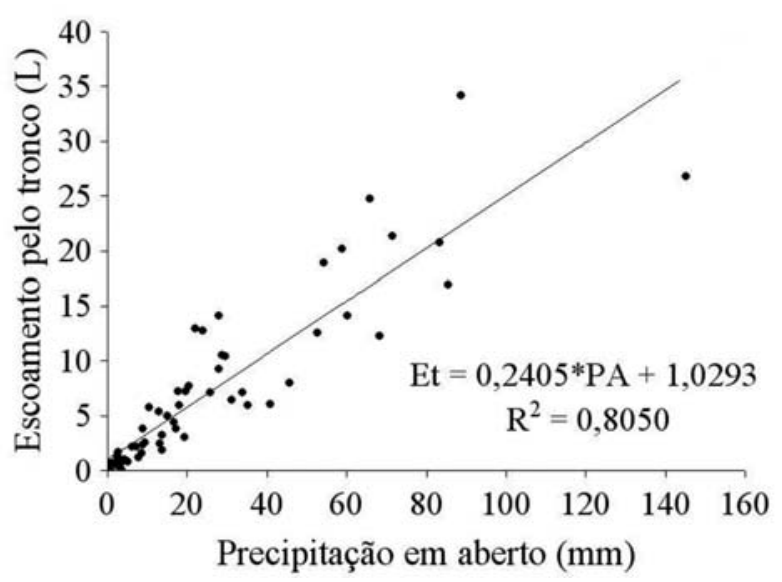

Figura 3 - Relação entre a precipitação em aberto (PA) e o escoamento pelo tronco da Euterpe edulis. Mata do Paraíso, Viçosa-MG, 2009-2011.

Figure 3 - Relationship between gross precipitation (GP) and stem flow of Euterpe edulis. Mata do Paraíso, Viçosa-MG, 2009-2011.

Para a bacia hidrográfica, o escoamento pelo tronco é irrelevante se comparado com a quantidade de água da chuva que chega ao solo via precipitação interna. Por isso, nos estudos de precipitação efetiva, muitos autores desprezam essa variável. Contudo, conforme mostrado neste trabalho, para alguns indivíduos o escoamento pelo tronco é de grande importância, dado o volume de água da chuva que chega à base do tronco de algumas espécies.

\section{CONCLUSÃO}

Com base nos resultados deste estudo, pode-se concluir que o escoamento pelo tronco representa, para o indivíduo, ganho significativamente elevado de água próximo às raízes. A Euterpe edulis foi a espécie com maior escoamento pelo tronco, apresentando, durante todo o período analisado, um volume médio escoado pelo tronco de 637,00 L.

No período analisado, não foi observado nenhum padrão com relação à qualidade da copa. As espécies com maior escoamento pelo tronco estão no estrado inferior e médio da floresta, sugerindo que essas espécies possuem algum mecanismo morfológico de captação de água da chuva.

Devido ao elevado volume de água direcionada ao solo via escoamento pelo tronco, algumas espécies possuem grande potencial na recuperação de áreas degradadas, podendo ser essa uma característica a ser considerada na escolha das espécies. Além disso, deve-se ressaltar a importância dessas espécies na prestação de serviços ambientais, haja vista sua contribuição na manutenção dos recursos hídricos.

Maiores estudos devem ser realizados com o intuito de entender os mecanismos de captação de água da chuva pelas espécies florestais e identificar as espécies com maior potencial para preservação e recuperação ambiental.

\section{REFERÊNCIAS}

APG III. An update of the Angiosperm Phylogeny Group classification for the orders and families of flowering plants: APG III. Botanical Journal of the Linnean Society, v.161, n.2, p.105-121, 2009.

CASTRO, P.S.; VALENTE, O.F.; COELHO, D.T.; RAMALHO, R.S. Interceptação da chuva por mata natural secundária na região de Viçosa MG. Revista Árvore, v.7, n.1, p.76-89, 1983.

CORREA, G. F. Modelo de evolução e mineralogia da fração argila de solos do planalto de Viçosa, MG. 1984. 87f. Dissertação (Mestrado em Solos e Nutrição de Plantas) - Universidade Federal de Viçosa, Viçosa, MG, 1984.

CROCKFORD, H.; RICHARDSON, D. P. Factors affecting the stemflow yield of a dry sclerophyll eucalypt forest, a Pinus radiata plantation and individual trees within the forest. Canberra: CSIRO, 1987. 27p.

GERMER, S.; ELSENBEER, H.; MORAES, J. M. Throughfall and temporal trends of rainfall redistribution in an open tropical rainforest, south-western Amazonia (Rondônia, Brazil).

Hydrology and Earth System Sciences, v.10, n.3, p.383-393, 2006.

HUBER, A. J.; OYARZÚN, C. O. Precipitacion neta e interceptacion en un bosque adulto de Pinus radiata (D. Don.). Bosque, v. 5, n. 1, p.13-20, 1983.

JOHNSSON, R. G. The interception, throughfall and stemflow in a forest in Highland Scotland

Revista Árvore, Viçosa-MG, v.39, n.3, p.423-430, 2015 
and the comparison with other upland forests in the U.K. Journal of Hydrology, v.118, n.1-4, p.281-287, 1990.

KELLMAN, M.; ROULET, N. Stemflow and throughfall in a tropical dry Forest. Earth Surface Processes Landforms, v.15, n.1, p.55-61, 1990.

LIMA, P. R. A.; LEOPOLDO, P. R. Interceptação de chuva por mata ciliar na região central do Estado de São Paulo. Energia na Agricultura, v.14, n.3, p.25-33, 1999.

LLOYD, C. R.; MARQUES, A. O. Spatial variability of throughfall and stemflow measurements 25 in Amazonian rainforest. Agricultural and Forest Meteorology, v.42, n.1, p.63-73, 1988.

OLIVEIRA JUNIOR, J. C. Precipitação

efetiva em Floresta Estacional

Semidecidual na reserva Mata do

Paraíso, Viçosa, Minas Gerais. 2006. $72 \mathrm{f}$.

Dissertação (Mestrado em Ciência Florestal) -

Universidade Federal de Viçosa, Viçosa, MG, 2006.

OLIVEIRA JUNIOR, J. C.; DIAS, H. C. T.

Precipitação efetiva em fragmento secundário da Mata Atlântica. Revista Árvore, v.29, n.1, p.9-15, 2005.
MANFROI, O.J.; KOICHIRO, K.; NOBUAKI, T.; MASAKAZU, S.; NAKAGAWA, M.; NAKASHIZUKA, T.; CHONG, L. The stemflow of trees in a Bornean lowland tropical forest. Hydrological Processes, v.18, n.13, p.2455-2474, 2004.

NÁVAR, J.; BRYAN, R. Interception loss and rainfall redistribution by three semi-arid growing shrubs in northeastern Mexico. Journal of Hydrology, v.115, n.1, p.51-63, 1990.

PRICE, N. W. A comparison of water balance components in natural and plantation forests in el Salvador, central America. Turrialba, v.32, n.4, p.399-416,1982.

SILVA JUNIOR, W. M.; MARTINS, S.V.; SILVA, A.F.; MARCO JÚNIOR, P. Regeneração de espécies arbustivo-arbórea em dois trechos de uma Floresta Estacional Semidecidual, Viçosa, MG. Scientia Forestalis, n.66, p.169-179, 2004.

TUCCI, C. E. M. Hidrologia: ciência e aplicação. 2.ed. Porto Alegre: UFGRS, 2001. 943p.

VELOSO, H. P.; RANGEL FILHO, A. L. R.; LIMA, J. C. A. Classificação da vegetação brasileira, adaptada a um sistema universal. Rio de Janeiro: IBGE, Departamento de Recursos Naturais e Estudos Ambientais, 1991. 